\title{
LOW MEMORY WAVELET-BASED HYPERSPECTRAL IMAGE CODING USING 2D DYADIC WAVELET TRANSFORM
}

\author{
Shrish Bajpai \\ Electronics and Communication Engineering Department, Faculty of Engineering, \\ Integral University, Lucknow, Uttar Pradesh, India \\ Naimur Rahman Kidwai \\ Electronics and Communication Engineering Department, Faculty of Engineering, \\ Integral University, Lucknow, Uttar Pradesh, India \\ Vishal Singh Chandel \\ Department of Applied Science and Humanities, Rajkiya Engineering College, \\ Ambedkar Nagar, Uttar Pradesh, India
}

\begin{abstract}
The proposed hyperspectral image compression algorithm employs $2 D$ dyadic wavelet transform to transform spectral frames and encode each plane using the $3 D$ Low Memory Block Tree Coding algorithm starting from the higher bit plane to the lower bit plane. The 3D dyadic wavelet transform which employees a significant memory in the transformation process, posing a challenge to the resource constraint hyperspectral image sensors. The results show that the proposed algorithm requires a low memory while maintaining the coding gain and complexity at par to the $3 D$ wavelet transform based hyperspectral image compression algorithms.
\end{abstract}

Key words: Wavelet Transform, Image Coding, Hyperspectral Image, Compression Algorithm, Transform Based Compression.

Cite this Article: Shrish Bajpai, Naimur Rahman Kidwai and Vishal Singh Chandel, Low Memory Wavelet-Based Hyperspectral Image Coding Using 2D Dyadic Wavelet Transform. International Journal of Electrical Engineering and Technology, 11(6), 2020, pp. 25-33.

http://iaeme.com/Home/issue/IJEET?Volume $=11 \&$ Issue $=6$

\section{INTRODUCTION}

In the last couple of decades, there is significant growth in computational technology. These computer-based machines generate a lot of data, and this data has been sent to the wireless channel. According to the report by CISCO, the mobile data has been reached up to 11.22 
Exabytes in 2017, which is around fivefold data growth as on 2012. It has been noted that the data is growing at a rate of $66 \%$ yearly [1-2].

Computer vision is one of the fastest-growing fields of computer science that has various applications in agriculture, automotive, banking, healthcare, remote sensing, security, etc [3]. The computer vision technology had been developed from the grayscale image to the hyperspectral (HS) image during a couple of decades. The single HS image is the collection of the hundreds of images that are taken for the electromagnetic spectrum from visible range to infrared range. It has been known that a single HS image can capture the fine detail of the elements present in the scene. This vast image data is a challenge for any HS image sensor. So, the HS image compression becomes a necessary process to save the sensor memory and energy, to increase its performance (efficiency), and reduce the data bandwidth with transmission time [4]. There are two types of redundancy exist in the HS images, namely spectral redundancy and spatial redundancy. The spectral redundancy exists due to the correlation between the two consecutive frames of HS image while the spatial residency exists due to the spatial variation of the pixels in the frame. The high correlation exists between the continuous frames of HS images that need to be exploited by reducing the redundancy [5].

The HS image compression algorithms (HSICA) can be classified into two different categories on the basis of data loss and coding process. Based on data loss, the HSICAs can be subdivided into the two groups named as lossy HSICAs and lossless HSICAs [6] while in the view of coding process of the algorithm, HSICA can be divided into three different groups called as the predictive coding, the vector quantization, and the transform coding [7].

The transform-based HS image coding algorithms are one of the most popular HS image coding algorithms that enable the medium complexity with high coding gain and flexible to the definition of hyperspectral image quality policies [8]. Meanwhile, in recent years, there is a rise in the transform coding algorithm for HS images, which includes explicitly transformbased set partition coding algorithms. Set partition coding algorithms can achieve a state of art performance with the embedded nature. The set partitioning algorithms can be categorized on the basis of partitioning rule or list implementation. On the basis of the partitioning rule, it can be subdivided into the three subcategories named as block cube partitioning (3D-SPECK and 3D-LSK), tree partitioning (3D-SPIHT and 3D-NLS) and block cube tree partitioning (3DWBTC and 3D-LMBTC). While for the list implementation, it can be divided into two subcategories named as having lists (3D-SPECK 3D-SPIHT and 3D-WBTC) or having no list (3D-LSK, 3D-NLS, and 3D-LMBTC) [9]. In the block cube partitioning, the whole HS image data is divided into the eight continuous block cubes, and each block cube is tested for the significance. If any element in the block cube is significant, then the whole block cube is tested and it is divided into 8 sub-blocks, and the process is repeated till it reaches to the single coefficient level, while for the tree partitioning, the whole HS image data is partitioning into the spatial orientation trees form in which the significance of the coefficient has been tested, and if any coefficient found significant, then the whole tree will be tested for significance. In the block cube tree set partitioning algorithms, the whole HS image data is partitioned into the continuous coefficient block cubes, and then block cube trees are formed with the root in the topmost subband. An algorithm that uses the list to track the coefficients is called the list based set partitioning HS image compression scheme while the compression algorithm uses the markers for tables to track the significance of the block cube or tree. The listless HS image compression algorithms are relatively fast, but they are memory consuming with reference to their list base versions [10].

The transform process and the coding process of the transform-based HSICA either the two stages run in parallel or sequentially. It has been observed that the $3 \mathrm{D}$ dyadic wavelet transform or any other 3D wavelet transform consumes a lot of memory, which needs to be 
reduced for the resource constrain HS image sensors. In this paper, we proposed a novel HSICA that reduces the transformed memory keeping the coding gain and complexity near to the 3D-DWT based 3D-LMBTC. The proposed algorithm is compared with the other state of art HSICAs on the basis of five metrics: the transformed memory, the coding memory, the peak signal to noise ratio (PSNR), the encoding time, and the decoding time taken by the proposed algorithm. The peak signal to noise ratio is a measurement of the distortion of the image, which is related to the compression ratio of the HS image. It also indicates the total quality of the reconstructed image with reference to the original image.

The paper is organized into four sections. The second section is dedicated to the proposed HSICA, which is adapted to the raw and processed HS images. The third section outlines the comparative results between the state of the art HSICAs with the proposed algorithm on the basis of the mentioned five parameters. Finally, the conclusions are given in Section IV.

\section{PROPOSED METHOD}

In the past few years, many significant efforts have been taken to reduce the memory requirement of the transform-based HS image coders. The memory requirement of the hyperspectral image order is divided into two parts transform memory and coding memory. The transform memory is a memory required for the wavelet transform and inverse wavelet transform, while the coding memory is the memory requirement for the encoding and decoding process of wavelet coefficients. Overall, any transform-based HSICA memory is either the sum of two memories or the maximum of both depending upon whether both stages run in parallel or they run in sequential form.

Dyadic Wavelet Transform: In this section, we have proposed the $2 \mathrm{D}$ dyadic wavelet transform based HS im-age compression algorithm, which is the low memory solution for the HS image. It reduces the memory requirement during the $2 \mathrm{D}$ wavelet transform due to the nature of transform with the cost of slightly higher complexity and slightly lower coding gain with reference to its $3 \mathrm{D}$ version. The traditional 3D dyadic wavelet transform requires a large memory, while the 2D dyadic wavelet transform needs less memory for the computation of the wavelet coefficients [9-11].

3D Low Memory Block Tree Coding (3D-LMBTC) : The 3D-Low Memory Block Tree Coding (3D-LMBTC) is a transform-based set partitioned HSICA that is an appropriate candidate for the low memory HS image sensors [9]. It is a listless version of 3D-Wavelet Block Tree Coding (3D-WBTC), which uses the excellent features of block cube-based algorithm and tree-based algorithm for an HS image. The algorithm uses four different types of markers for the tracking of the significance of the coefficients and sets, which reduce the complexity and coding memory of the compression scheme. The significance testing of the block cube and block cube tree with the help of equation 1 and equation 2 . For the $\mathrm{nt}^{\mathrm{h}}$ bit plane, the threshold is given as $\mathrm{T}=2^{\mathrm{n}}$.

$$
\begin{aligned}
& S_{n}(B)=\left\{\begin{array}{cc}
1 & \text { if } T \leq \max _{i \in B}\left[\left|C_{i}\right|\right] \leq 2 T \\
0 & \text { if } \max _{i \in B}\left[\left|C_{i}\right|\right]<T \\
N u l l & \text { if } \max _{i \in B}\left[\left|C_{i}\right|\right] \geq 2 T
\end{array}\right.
\end{aligned}
$$

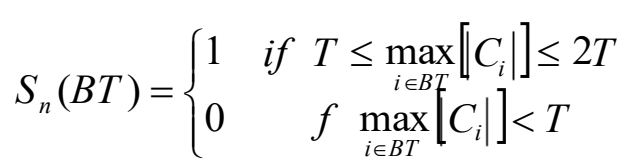




\section{EXPERIMENTAL RESULTS AND ANALYSIS}

All transform-based set partitioned HSICAs are applied to the same computer platform having the working memory of $8 \mathrm{~GB}$ and 4 th generation i3 processor on the numerical computing simulation software. The two raw images (Yellowstone uncalibrated scene 3 and Yellowstone uncalibrated scene 10) have been taken for the analysis having a dimension of 128 for each dimension. The HS image data can be seen as the three dimensions of $\mathrm{x}, \mathrm{y}$, and $\mathrm{z}$, where $\mathrm{x}$ and $y$ are the spatial dimensions and $z$, is known as a spectral dimension of the HS image, which corresponds to the different wavelengths [9]. The HS images are transformed dyadic wavelet transform (3D or 2D) as mentioned, and then it's floating-point transform coefficients are quantized to the nearest integer. After that, it reads into a linear index array which is the onedimensional matrix through the Morton mapping [6].

Coding Efficiency : The coding efficiency is a measurement of rate-distortion performance of any HS image compression algorithm, which is measured in terms of bit per pixel per band to achieve a minimum desire quality of the reconstructed HS image. The peak signal to noise ratio is a parameter which measures the quality of the reconstructed HS image is defined mathematically [12] in equation 3
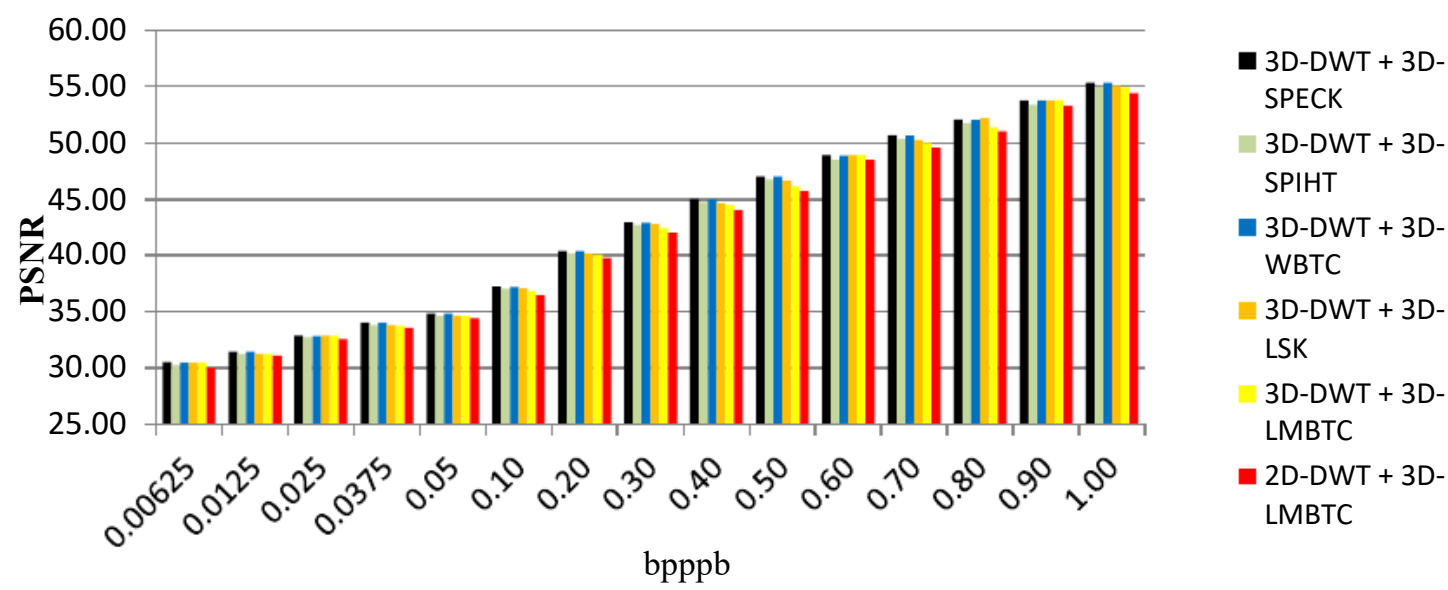

Figure 1 (a) Peak Signal to Noise Ratio of the Yellowstone uncalibrated scene 3 for fifteen different bit rates.
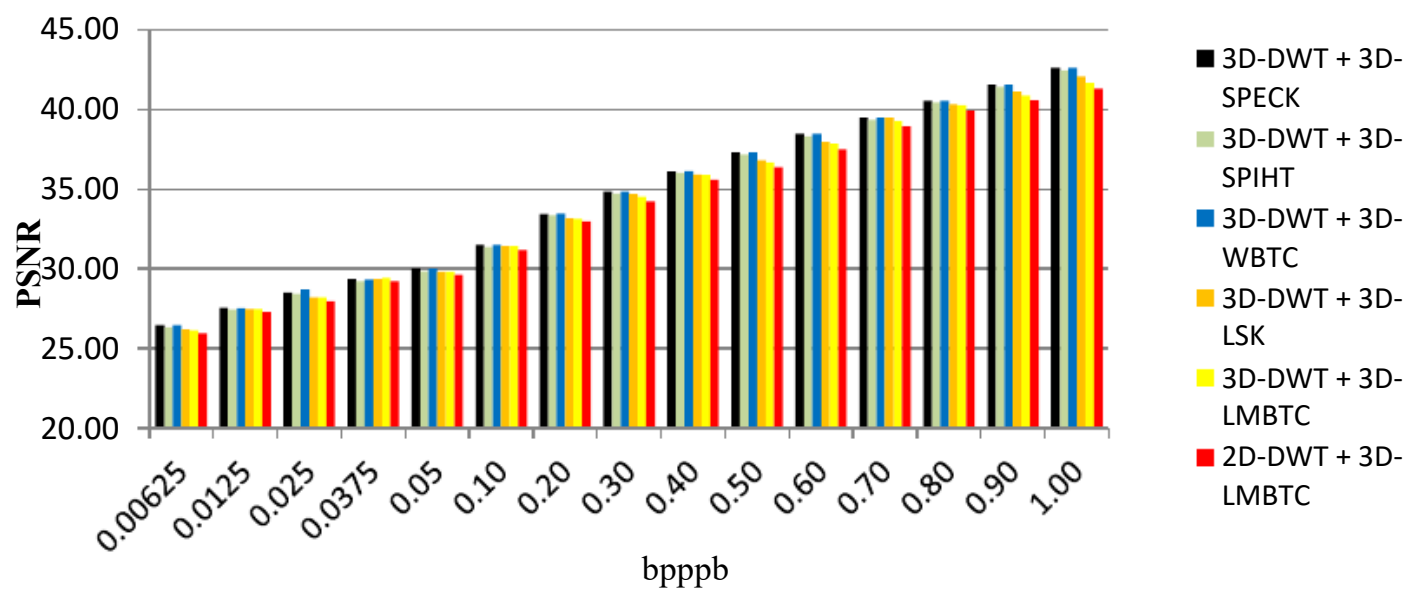

Figure 1 (b) Peak Signal to Noise Ratio of the Yellowstone uncalibrated scene 10 for fifteen different bit rates. 


$$
P S N R=10 \log _{10}\left[\frac{\text { Maximum Signal Power }}{M S E}\right]
$$

where MSE is a mean square error of the reconstructed HS image [13-14] defined in equation 4

$$
M S E=\frac{1}{N_{p i x}} \sum_{x, y, z}[f(x, y, z)-g(x, y, z)]^{2}
$$

$\mathrm{N}_{\text {pix }}$ is a total number of pixels in all frames for each HS image. The $f(x, y, z)$ is the original HS image, and $\mathrm{g}(\mathrm{x}, \mathrm{y}, \mathrm{z})$ is the reconstructed HS image.

It has been observed that the coding train gain been reduced from $0.1 \mathrm{~dB}$ to $0.5 \mathrm{~dB}$ at different bit rates, as shown in Fig 1 (a) and Fig 1 (b) with increased complexity due to application of the 2D-DWT frame by frame. However, at the low bit rates below $0.2 \mathrm{bpppb}$ (bit per pixel per band) [9], the proposed algorithm works at par with 3D- DWT based 3DLMBTC. Fig 1 (a) and Fig 1 (b) are the comparative analysis of proposed algorithm with the 3D dyadic wavelet transform based 3D-SPECK [15], 3D-SPIHT [16], 3D-LSK [17], 3DWBTC [6] and 3D-LMBTC [9].

Computation Memory : Table 1 gives a comparative analysis of the different set partitioning based HSICAs. It has been observed that the proposed scheme is using the same memory as the 3D-DWT based 3D-LMBTC, and it is fixed, which is dependent on the size of the HS image. Due to the listless approach, it requires a fix size memory, which is depended on the size of HS image. It needs a fixed size memory of $12 \mathrm{~KB}$ for the tracking of the sets and the coefficients.

Table 2 gives the comparative analysis of working memory required by five-level of 2D dyadic wavelet transform and 3D dyadic wavelet transform. It has been clearly observed that there is a multiple fold increase in the consumption of the memory of 3D-DWT compare to 2D-DWT. This is because that the 2D dyadic wavelet transform requires less memory because it applied to the two dimensions only, while the 3D dyadic wavelet transform is applied to the three-dimension which increases the size and more computational memory is needed to calculate the wavelet coefficients.

Complexity Analysis : The computational complexity of the proposed HSICA is calculated by estimating the computation time required for the encoding of wavelet transform coefficients and for decoding the generated bit-stream at the given bitrate. The complexity of the proposed HS image compression is compared with other states of the art HS image compression algorithms in table 3 and table 4 for the different bit rates. From table 3 and table 4 , it is observed that for each algorithm, the encoding and decoding time increases as the bit rate increases. This is an expected outcome because more coefficients needed to be encoded, and hence the encoding time as well as decoding time increases as the bit rate increases.

It has also been evident from Table 3 and Table 4 that the listless coding algorithms have lower computation time than their corresponding list base coding algorithms. The list base coding algorithms use the linked list to keep track of the transform coefficients and sets, and it will grow exponentially with the increase bit rate. The management and processing of these linked lists need higher computational demands, and it has longer computational time. The listless algorithms use the fixed-size state tables instead of a linked list. These fix size state tables do not require any memory or operational management; therefore, they require less time for the encoding of the transform coefficients. The proposed algorithm is a variant of 3D-LMBTC, which uses the four types of markers for the tracking of the coefficients. 
Low Memory Wavelet-Based Hyperspectral Image Coding Using 2D Dyadic Wavelet Transform

Table 1 Comparative Analysis of memory consumption by the different HSICAs at the coding stage

\begin{tabular}{|c|c|c|c|c|c|c|c|c|c|c|c|c|}
\hline \multicolumn{7}{|c|}{$\begin{array}{l}\text { Memory of Yellowstone uncalibrated scene } 3 \text { for the different } \\
\text { compression scheme }\end{array}$} & \multicolumn{6}{|c|}{$\begin{array}{c}\text { Memory of Yellowstone uncalibrated scene for the } \\
\text { different compression scheme }\end{array}$} \\
\hline 을 & 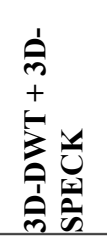 & 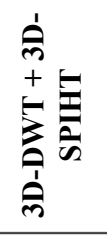 & 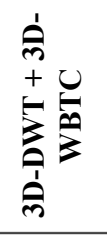 & 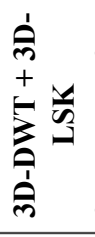 & 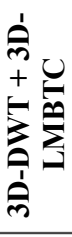 & 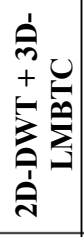 & 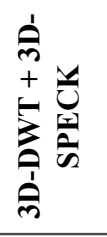 & $\begin{array}{l}\dot{1} \\
+ \\
+ \\
\hat{n} \\
\hat{n}\end{array}$ & $\begin{array}{l}\dot{1} \\
\text { ê } \\
+ \\
1 \\
\text { ê }\end{array}$ & 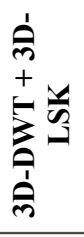 & $\begin{array}{l}\dot{1} \\
\text { लिu } \\
+0 \\
\hat{n}^{\circ}\end{array}$ & 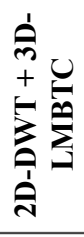 \\
\hline 0.00625 & 16.3 & 16.9 & 8.2 & 512 & 12 & 12 & 19.1 & 14.7 & 11.2 & 512 & 12 & 12 \\
\hline 0.0125 & 25.9 & 28.4 & 20.1 & 512 & 12 & 12 & 33.3 & 37.3 & 34.3 & 512 & 12 & 12 \\
\hline 0.025 & 71.1 & 73.0 & 62.6 & 512 & 12 & 12 & 64.6 & 71.4 & 64.2 & 512 & 12 & 12 \\
\hline 0.0375 & 78.2 & 80.7 & 71.6 & 512 & 12 & 12 & 111.8 & 115.6 & 101.1 & 512 & 12 & 12 \\
\hline 0.05 & 116.9 & 124.1 & 109.4 & 512 & 12 & 12 & 162.8 & 123.3 & 113.3 & 512 & 12 & 12 \\
\hline 0.1 & 205.4 & 204.1 & 185.6 & 512 & 12 & 12 & 276.4 & 288.7 & 281.5 & 512 & 12 & 12 \\
\hline 0.2 & 396.9 & 413.7 & 378.1 & 512 & 12 & 12 & 447.9 & 489.0 & 446.0 & 512 & 12 & 12 \\
\hline 0.3 & 677.9 & 682.5 & 621.2 & 512 & 12 & 12 & 822.3 & 832.6 & 826.1 & 512 & 12 & 12 \\
\hline 0.4 & 725.9 & 752.6 & 708.4 & 512 & 12 & 12 & 919.9 & 881.2 & 891.4 & 512 & 12 & 12 \\
\hline 0.5 & 947.4 & 946.7 & 894.3 & 512 & 12 & 12 & 1038.4 & 1055.3 & 1036.6 & 512 & 12 & 12 \\
\hline 0.6 & 1119.2 & 1155.4 & 1090.9 & 512 & 12 & 12 & 1352.1 & 1388.6 & 1355.1 & 512 & 12 & 12 \\
\hline 0.7 & 1234.2 & 1243.4 & 1120.9 & 512 & 12 & 12 & 1633.8 & 1561.4 & 1592.3 & 512 & 12 & 12 \\
\hline 0.8 & 1343.5 & 1323.5 & 1282.4 & 512 & 12 & 12 & 1681.0 & 1652.4 & 1633.6 & 512 & 12 & 12 \\
\hline 0.9 & 1439.0 & 1473.2 & 1400.1 & 512 & 12 & 12 & 1741.3 & 1709.9 & 1712.3 & 512 & 12 & 12 \\
\hline 1 & 1498.0 & 1517.2 & 1440.1 & 512 & 12 & 12 & 1825.2 & 1813.1 & 1824.5 & 512 & 12 & 12 \\
\hline
\end{tabular}

Table 2 Comparative Analysis of memory used by in transform stage

\begin{tabular}{l|l}
\hline \hline \multicolumn{2}{c}{ Memory required for the Transform (5 Level) } \\
\hline 3D Dyadic Wavelet Transform & $38.346 \mathrm{MB}$ \\
2D Dyadic Wavelet Transform & $\mathbf{3 4 8 . 9 9 1 ~ K B}$ \\
\hline \hline
\end{tabular}

Table 3 Comparative Analysis of encoding time consumed by the different HSICAs

\begin{tabular}{|c|c|c|c|c|c|c|c|c|c|c|c|c|}
\hline \multicolumn{7}{|c|}{$\begin{array}{c}\text { Encoding Time of Yellowstone uncalibrated scene } 3 \text { for the } \\
\text { different compression scheme }\end{array}$} & \multicolumn{6}{|c|}{$\begin{array}{l}\text { Encoding Time of Yellowstone uncalibrated scene } 10 \\
\text { for the different compression scheme }\end{array}$} \\
\hline 을 & 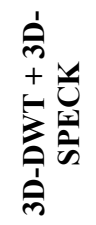 & 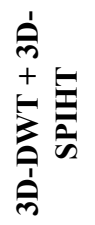 & $\begin{array}{l}\dot{1} \\
\text { mे } \\
+ \\
\text { ले } \\
\text { ले }\end{array}$ & 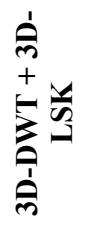 & 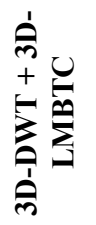 & 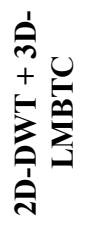 & 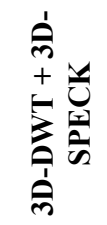 & 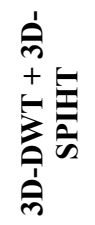 & $\begin{array}{l}\text { ले } \\
+0 \\
\text { ले }\end{array}$ & 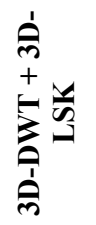 & 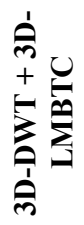 & 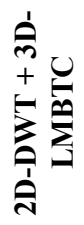 \\
\hline 0.00625 & 0.9 & 1.0 & 0.5 & 0.7 & 0.4 & 0.7 & 1.8 & 0.6 & 0.8 & 0.3 & 0.9 & 1.1 \\
\hline 0.0125 & 1.6 & 1.1 & 1.3 & 0.4 & 1.1 & 1.4 & 2.5 & 1.1 & 1.4 & 0.4 & 1.2 & 1.4 \\
\hline 0.025 & 2.9 & 1.6 & 1.8 & 0.5 & 1.4 & 1.8 & 5.0 & 1.5 & 1.7 & 0.4 & 1.5 & 1.6 \\
\hline 0.0375 & 5.0 & 2.2 & 2.4 & 0.5 & 1.7 & 2.1 & 5.1 & 2.2 & 2.1 & 0.5 & 1.7 & 1.9 \\
\hline 0.05 & 6.7 & 4.3 & 2.7 & 0.6 & 2.1 & 2.5 & 8.5 & 3.3 & 3.5 & 0.6 & 2.0 & 2.2 \\
\hline 0.1 & 18.2 & 9.9 & 7.1 & 0.8 & 3.2 & 3.7 & 18.8 & 8.0 & 5.9 & 0.7 & 3.0 & 3.3 \\
\hline 0.2 & 57.3 & 24.3 & 25.0 & 1.2 & 5.5 & 5.9 & 83.5 & 29.0 & 23.5 & 1.0 & 5.0 & 5.4 \\
\hline 0.3 & 97.1 & 39.9 & 47.9 & 1.4 & 6.7 & 8.2 & 110.2 & 50.3 & 32.5 & 1.3 & 6.6 & 7.7 \\
\hline 0.4 & 205.5 & 104.1 & 122.3 & 1.8 & 9.3 & 10.3 & 326.5 & 181.1 & 198.7 & 1.7 & 9.2 & 10.1 \\
\hline 0.5 & 302.7 & 130.9 & 176.7 & 2.2 & 10.5 & 11.7 & 471.9 & 241.5 & 294.1 & 1.9 & 11.5 & 11.8 \\
\hline
\end{tabular}


Shrish Bajpai, Naimur Rahman Kidwai and Vishal Singh Chandel

\begin{tabular}{c|cccccc|cccccc}
\hline \hline 0.6 & 348.8 & 140.5 & 190.4 & 2.5 & 11.5 & 13.1 & 588.3 & 263.7 & 363.2 & 2.2 & 11.3 & 12.8 \\
0.7 & 706.4 & 337.8 & 528.3 & 2.7 & 14.3 & 15.2 & 678.3 & 281.3 & 398.3 & 2.5 & 12.5 & 13.8 \\
0.8 & 835.1 & 408.4 & 601.1 & 3.0 & 15.7 & 17.2 & 1150.8 & 531.0 & 771.8 & 2.9 & 15.8 & 17.2 \\
0.9 & 980.8 & 414.4 & 633.2 & 3.4 & 16.8 & 18.4 & 1745.2 & 883.0 & 1223.2 & 3.1 & 17.1 & 18.9 \\
1 & 1372.9 & 632.4 & 976.9 & 3.7 & 19.9 & 21.1 & 2557.6 & 1034.1 & 1517.0 & 3.3 & 18.3 & 20.2 \\
\hline \hline
\end{tabular}

Table 4 Comparative Analysis of decoding time consumed by the different HSICAs

\begin{tabular}{|c|c|c|c|c|c|c|c|c|c|c|c|c|}
\hline \multicolumn{7}{|c|}{$\begin{array}{l}\text { Decoding Time of Yellowstone uncalibrated scene } 3 \text { for the } \\
\text { different compression scheme }\end{array}$} & \multicolumn{6}{|c|}{$\begin{array}{l}\text { Decoding Time of Yellowstone uncalibrated scene } \\
10 \text { for the different compression scheme }\end{array}$} \\
\hline & $\begin{array}{l}\text { ले } \\
+\frac{1}{4} \\
5 \text { ज्ञ } \\
\text { ले }\end{array}$ & $\begin{array}{l}\text { ले } \\
+ \\
\text { ले } \\
\text { ले }\end{array}$ & 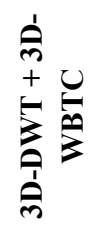 & $\begin{array}{l}\dot{1} \\
+ \\
+ \\
\text { ले } \\
\text { ले }\end{array}$ & 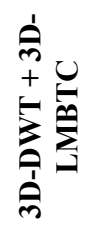 & 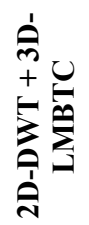 & 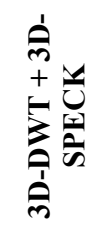 & $\begin{array}{l}\text { ले } \\
+ \\
5 \\
\text { ले } \\
\text { ले }\end{array}$ & $\begin{array}{l}\text { ले } \\
+0 \\
\text { के } \\
\text { ले }\end{array}$ & $\begin{array}{l}\dot{1} \\
+ \\
+ \\
-1 \\
\text { ले } \\
\text { ले }\end{array}$ & $\begin{array}{l}\dot{1} \\
+ \\
+ \\
5 \\
\text { ले }\end{array}$ & 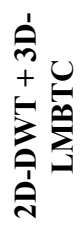 \\
\hline 0.00625 & 0.7 & 0.8 & 0.2 & 0.5 & 0.4 & 0.5 & 0.8 & 0.3 & 0.2 & 0.2 & 0.5 & 0.7 \\
\hline 0.0125 & 0.9 & 0.8 & 0.4 & 0.3 & 0.5 & 0.8 & 1.9 & 0.3 & 0.4 & 0.3 & 0.9 & 1.2 \\
\hline 0.025 & 1.6 & 1.2 & 0.6 & 0.4 & 0.7 & 1.0 & 2.3 & 0.7 & 0.7 & 0.4 & 0.7 & 1.5 \\
\hline 0.0375 & 3.5 & 1.5 & 1.2 & 0.4 & 0.9 & 1.3 & 2.8 & 1.2 & 0.9 & 0.4 & 0.8 & 1.7 \\
\hline 0.05 & 4.6 & 2.6 & 1.6 & 0.5 & 1.1 & 1.5 & 5.9 & 2.2 & 2.3 & 0.5 & 1.0 & 1.9 \\
\hline 0.1 & 14.5 & 6.6 & 9.5 & 0.7 & 1.9 & 2.2 & 12.8 & 6.5 & 4.4 & 0.6 & 1.8 & 2.4 \\
\hline 0.2 & 49.3 & 22.3 & 17.6 & 1.0 & 3.5 & 4.7 & 62.2 & 25.8 & 21.6 & 1.0 & 3.3 & 3.7 \\
\hline 0.3 & 81.9 & 31.6 & 40.0 & 1.4 & 5.1 & 7.1 & 80.0 & 47.3 & 29.2 & 1.2 & 4.9 & 6.2 \\
\hline 0.4 & 189.6 & 90.4 & 109.7 & 1.7 & 6.7 & 8.4 & 306.7 & 171.6 & 175.3 & 1.4 & 6.7 & 8.1 \\
\hline 0.5 & 284.1 & 117.7 & 155.8 & 2.0 & 8.1 & 10.5 & 441.3 & 218.6 & 262.2 & 1.7 & 7.6 & 9.4 \\
\hline 0.6 & 321.1 & 128.3 & 176.2 & 2.4 & 9.4 & 11.2 & 555.1 & 229.7 & 319.1 & 1.9 & 9.0 & 10.8 \\
\hline 0.7 & 684.0 & 308.8 & 576.7 & 2.5 & 11.0 & 13.7 & 609.3 & 246.5 & 358.5 & 2.2 & 10.5 & 12.1 \\
\hline 0.8 & 821.8 & 373.0 & 552.6 & 2.7 & 12.5 & 15.1 & 1080.0 & 496.2 & 717.3 & 2.5 & 11.8 & 14.4 \\
\hline 0.9 & 859.6 & 399.7 & 588.7 & 3.2 & 13.9 & 16.3 & 1692.5 & 817.9 & 1130.1 & 3.0 & 13.3 & 15.7 \\
\hline 1 & 1258.0 & 589.2 & 849.3 & 3.4 & 15.6 & 18.7 & 2179.1 & 929.9 & 1384.1 & 3.4 & 14.7 & 17.2 \\
\hline
\end{tabular}

The computational time for the 3D-LSK is less than 3D-LMBTC because there is one more step for the significance testing of block cubes, which lead to the increase in computational time. The proposed algorithm is taking more time than the original 3DLMBTC because of the use of a two-dimensional dyadic wavelet transform which is applied frame-by-frame; thus it increases time for encoding process and decoding process.

\section{CONCLUSION}

In order to meet the constraint of low-cost HS image sensors and associated multimedia devices, also a low memory image coder is an essential requirement. In this paper, we propose a low memory algorithm that is suitable for the memory constraint image sensors. The use of a two-dimensional dyadic wavelet transform, the transform memory is reduced significantly, and the memory used in the algorithm reduced by the 3D-LMBTC, which is the low memory HS image compression scheme. From the simulation result, it is observed that the $2 \mathrm{D}$ dyadic wavelet transform requires only 349-kilobyte memory. But it's 3D version utilize the $40 \mathrm{MB}$ memory, which is significantly large in multifold. So, the combination of applying $2 \mathrm{D}$ dyadic wavelet transform frame by frame and use of the 3D-LMBTC is the optimum solution for the low memory HS image sensors. 


\section{ACKNOWLEDGMENT}

One of the authors, Vishal Singh Chandel, is highly thankful to TEQIP-III scheme of Rajkiya Engineering College, Ambedkar Nagar for the financial support.

\section{REFERENCES}

[1] Sharma, Divya, Yogendra Kumar Prajapati, and Rajeev Tripathi. "Spectrally efficient 1.55 $\mathrm{Tb} / \mathrm{s}$ Nyquist-WDM super channel with mixed line rate approach using 27.75 Gbaud PMQPSK and PM-16QAM." Optical Engineering 57.7 (2018): 076102.

[2] Sharma, Divya, Y. K. Prajapati, and R. Tripathi. "Success Journey of Coherent PM-QPSK Technique with its Variants: A Survey." IETE Technical Review (2018): 1-20.

[3] Prabhu, N., Manoj K. Arora, and R. Balasubramanian. "Spatial Resolution Enhancement Mapping of Hyperspectral Image via Pixel Filling Algorithm." Journal of the Indian Society of Remote Sensing: 1-11.

[4] Chutia, Dibyajyoti, et al. "Hyperspectral remote sensing classifications: a perspective survey." Transactions in GIS 20.4 (2016): 463-490.

[5] Bajpai, Shrish, Harsh Vikram Singh, and Naimur Rahman Kidwai. "Feature extraction \& classification of hyperspectral images using singular spectrum analysis \& multinomial logistic regression classifiers." 2017 International Conference on Multimedia, Signal Processing and Communication Technologies (IMPACT). IEEE, 2017.

[6] Bajpai, Shrish, Naimur Rahman Kidwai, and Harsh Vikram Singh. "3D wavelet block tree coding for hyperspectral images." International Journal of Innovative Technology and Exploring Engineering (IJITEE) 8.6C (2019): 64-68.

[7] Dusselaar, Rui, and Manoranjan Paul. "Hyperspectral image compression approaches: opportunities, challenges, and future directions: discussion." JOSA A 34.12 (2017): 21702180.

[8] Penna, Barbara, et al. "Transform coding techniques for lossy hyperspectral data compression." IEEE Transactions on Geoscience and Remote Sensing 45.5 (2007): 14081421.

[9] Bajpai, S., Kidwai, N. R., Singh, H. V., \& Singh, A. K. "Low memory block tree coding for hyperspectral images." Multimedia Tools and Applications 78.19 (2019): 27193-27209.

[10] Fowler, James E., and Justin T. Rucker. "Three-dimensional wavelet-based compression of hyperspectral imagery." Hyperspectral Data Exploitation: Theory and Applications (2007): 379-407.

[11] Kidwai, Naimur Rahman, Ekram Khan, and Martin Reisslein. "ZM-SPECK: A fast and memoryless image coder for multimedia sensor networks." IEEE Sensors Journal 16.8 (2016): 2575-2587.

[12] Bhardwaj, Rupali. "Enhanced encrypted reversible data hiding algorithm with minimum distortion through homomorphic encryption." Journal of Electronic Imaging 27.2 (2018): 023017.

[13] Christophe, Emmanuel, Corinne Mailhes, and Pierre Duhamel. "Hyperspectral image compression: adapting SPIHT and EZW to anisotropic 3-D wavelet coding." IEEE Transactions on Image Processing 17.12 (2008): 2334-2346.

[14] Patra, Anirban, et al. "A Novel Approach to Compression of Satellite Images Using Butterworth Filtering." Information, Photonics and Communication. Springer, Singapore, 2020. 179-183. 
[15] Tang, Xiaoli, and William A. Pearlman. "Lossy-to-lossless block-based compression of hyperspectral volumetric data." 2004 International Conference on Image Processing, 2004. ICIP'04.. Vol. 5. IEEE, 2004.

[16] Tang, Xiaoli, Sungdae Cho, and William A. Pearlman. "3D set partitioning coding methods in hyperspectral image compression." Proceedings 2003 International Conference on Image Processing (Cat. No. 03CH37429). Vol. 2. IEEE, 2003.

[17] Ngadiran, Ruzelita, et al. "Efficient implementation of 3D listless SPECK." International Conference on Computer and Communication Engineering (ICCCE'10). IEEE, 2010. 